\title{
Prediction of Ignition Regimes in DME/Air Mixtures with Temperature and Concentration Fluctuations
}

\author{
Minh Bau Luong *, Francisco E. Hernández Pérez ${ }^{\dagger}$, Aliou Sow ${ }^{\ddagger}$, and Hong G. Im ${ }^{\S}$ \\ King Abdullah University of Science and Technology (KAUST), Thuwal 23955-6900, Saudi Arabia
}

The objective of the present study is to establish a theoretical prediction of the autoignition behavior of a reactant mixture for a given initial bulk mixture condition. The ignition regime criterion proposed by Im and coworkers based on the Sankaran number (Sa), which is a ratio of the laminar flame speed to the spontaneous ignition front speed, is extended to account for both temperature and equivalence ratio fluctuations. The extended ignition criterion is then applied to predict the autoignition characteristics of dimethyl ether (DME)/air mixtures and validated by two-dimensional direct numerical simulations (DNS). The response of the ignition mode of DME/air mixtures to three initial mean temperatures of $770,900 \mathrm{~K}$, and $1045 \mathrm{~K}$ lying within/outside the NTC regime, two levels of temperature and concentration fluctuations at a pressure of $30 \mathrm{~atm}$ and equivalence ratio of 0.5 is systematically investigated. The statistical analysis is performed, and a newly developed criterion -the volumetric fraction of $\mathrm{Sa}<\mathbf{1 . 0}$, $F_{\mathrm{S}, S}$, is proposed as a deterministic criterion to quantify the fraction of heat release attributed to strong ignition. It is found that the strong and weak ignition modes are well captured by the predicted $S a$ number and $F_{\mathrm{Sa}, S}$ regardless of different initial mean temperatures and the levels of mixture fluctuations and correlations. $\mathrm{Sa}_{p}$ and $F_{\mathrm{Sa}, S}$ demonstrated under a wide range of initial conditions as a reliable criterion in determining a priori the ignition modes and the combustion intensity.

\section{Nomenclature}

$\begin{array}{ll}\text { DNS } & \text { Direct numerical simulation } \\ \text { SI (WI) } & \text { Strong (weak) ignition } \\ \text { PDF } & \text { Probability density function } \\ \text { NC (UC) } & \text { Negatively-correlated (uncorrelated) } \\ T, p, \text { and } \phi & \text { Temperature, pressure, and equivalence ratio } \\ T_{0}, p_{0}, \text { and } \phi_{0} & \text { Initial mean } T, p \text {, and } \phi \\ \text { RMS } & \text { Root mean square } \\ T^{\prime} \& \phi^{\prime} & \text { RMS of } T \text { and } \phi \\ u^{\prime} & \text { Turbulent velocity fluctuation } \\ \text { Sa } & \text { Sankaran number } \\ \text { Sa } & \text { Predicted Sankaran number } \\ \overline{S a}_{F_{S a}} & \text { Mean Sa } \\ F_{S} & \text { Volumetric fraction of Sa }<1 \\ \tau_{i g} & \text { HRR fraction by the spontaneous ignition } \\ \tau_{t}\left(\tau_{f}\right) & \text { Ignition delay time } \\ S_{L} & \text { Turbulent (flame) time scale } \\ S_{s p} & \text { Laminar flame speed } \\ l_{e}, l_{T}, l_{\phi} & \text { Spontaneous front speed } \\ \mathrm{Da} & \text { Integral length scale of turbulence, temperature, equivalence ratio } \\ & \text { Damköhler number }\end{array}$

\footnotetext{
*Postdoctoral fellow, Clean Combustion Research Center, KAUST

${ }^{\dagger}$ Research scientist, Clean Combustion Research Center, KAUST

$\pitchfork$ Postdoctoral fellow, Clean Combustion Research Center, KAUST

${ }^{\S}$ Professor of Mechanical Engineering, Clean Combustion Research Center, KAUST, Associate Fellow
} 


\section{Introduction}

Advanced low-temperature-combustion (LTC) strategies of downsized and boosted engines offer ultra-low emissions and higher efficiencies [1-8]. The combustion is chemically driven by spontaneous autoignition process with no direct means to control the ignition timing and combustion rate. The autoignition process and its timing are highly sensitive to the fuel types and operating conditions including the intake temperature and pressure, thermal and compositional non-uniformities of fuel/air mixture, the amount of EGR, cooling temperature. As such, this engine types have issues of vulnerable pre-ignition, a higher possibility of knock, even super-knock characterized by high-pressure peaks and oscillations, which may damage the engine block [9-16]. Therefore, a reliable theory to predict such abnormal ignition phenomena is of critical importance [9, 17,-19].

To reduce an excessive heat rise rate (HRR), some levels of thermal and compositional fluctuations are generated to achieve a smooth combustion process [1-4]. In the presence of thermal and/or concentration fluctuations, $T^{\prime}$ and $\phi^{\prime}$, a mixed combustion mode of spontaneous ignition and deflagration co-exists, which helps prolong the combustion duration, alleviate the fuel consumption rate and lower the peak pressure rise rate (PRR) [20-55].

From the displacement speed and Damköhler number analyses, Yoo et al. [25] found that in general, larger $T^{\prime}$ induces greater temporal distribution of the mean HRR attributed to the predominant deflagration mode at the reaction fronts for large $T^{\prime}[20-22,25,-27,29,34,53]$. However, this effect is significantly reduced when the initial mean temperature, $T_{0}$, falls within the NTC regime the ignition delay time becomes insensitive to the temperature variations. It was found that larger length scale and/or $T_{0}$ in the NTC regime induce a greater amplitude of the PRR oscillation, and the ignition-front speed of the cases inside the NTC regime travels faster, leading to more interactions between the pressure wave and the developing reaction fronts, which in turn facilitates the formation of detonation wave [54, 55, 55, 60].

In a DNS study, Sankaran and coworkers found that the autoignition of $\mathrm{H}_{2}$ /air mixture with different $T^{\prime}$ was accurately predicted by a nondimensional Sankaran number, Sa, which is proportional to the ratio of laminar flame speed to the thermal gradient characterized spontaneous propagation speed [20]. In both experimental and computational studies, Sa was demonstrated as an accurate predictive criterion of the strong/weak mode for homogeneous mixtures in the presence of thermal non-uniformities [61-65]. Strozzi et al. reported a satisfactory agreement between a quantitative Sa-based prediction of the occurrence of autoignition fronts and deflagrations and the chemiluminescence images in an RCM during the early and intermediate stages of combustion [61]. Mansfield et al. conducted experimental studies to investigate the ignition behavior of syngas and iso-octane within a rapid compression facility (RCF) [62 64]. They found that the Sa number accurately predicts the location of strong ignition limit a priori for various equivalence ratios and locations.

Inspired by the Sa-based criterion, Im et al. performed scaling analysis to take into account the turbulent effect on the ignition process and derived the turbulent ignition regime diagram to identify whether a combustion process is ignition controlled or flame-propagation controlled [19]. The ignition regimes were classified into three categories: (1) weak ignition with the dominant mode of deflagration, (2) reaction-dominant strong, and (3) mixing-dominant strong with the dominant mode of spontaneous ignition. A recent DNS study has been conducted to verify the ignition regime diagram using syngas [66]. The results were consistent with the predictions by the ignition regime diagram. Grogan et al. also proposed an alternative ignition regime diagram specifically applicable to rapid compression machines [18].

Previous studies were mainly focused on the fuels with a single-stage ignition. As such, more validations are needed to verify the prediction of the ignition criterion [19] for complex fuels exhibiting the NTC behavior over a wide range of temperature, and for different $T_{0}$ and levels of temperature and concentration fluctuations. In addition, deflagrations and autoignition fronts were observed during the subsequent combustion processes, so quantifying their respective contributions to the heat release is desirable. The objective of the present study is twofold: (1) to extend the ignition regime diagram proposed by Im et al. [19] to accommodate both temperature and concentration fluctuations, and further validated by a NTC fuel by varying $T_{0}, T^{\prime}, \phi^{\prime}$, and $T-\phi$ correlations, and (2) to predictively quantify the combustion intensity attributed from the spontaneous ignition.

\section{Methodology}

\section{A. Ignition regime diagram with temperature fluctuations}

In this section, the turbulent ignition regime diagram proposed by Im et al. [19] is briefly summarized. The regime is further extended to apply for both temperature and concentration fluctuations [16]. The turbulent ignition regime diagram was inspired by the nondimensional Zeldovich-Sankaran criterion. According to Zeldovich's theory [67], the 
spontaneous propagation speed of an ignition front, $S_{s p}$, is expressed as

$$
S_{s p}=\left|\nabla \tau_{i g}\right|^{-1}
$$

where $\tau_{i g}$ is the homogeneous ignition delay. If $S_{s p}$ is sufficiently large and comparable to the speed of sound, the combustion wave may be coupled with the acoustic wave, leading to rapid pressure-rise and in some circumstances a detonation. If there is almost homogeneity (nearly zero gradient), $S_{s p}$ approaches infinity leading to negligible interactions between the reaction front and pressure wave. In such scenario, thermal explosion is expected to occur as spontaneous ignition mode, which characterized by extremely high pressure rise rate resulting from the volumetric autoignition [9, 67]. If there is a large temperature gradient, pressure wave attenuates such that flame front propagation or deflagration is the controlled mode.

Based on the two characteristic speeds of the laminar flame speed, $S_{L}$, and the spontaneous ignition-front speed, Sankaran et al. [20] proposed a nondimensional number, Sa, as

$$
\mathrm{Sa}=\beta \frac{S_{L}}{S_{s p}},
$$

where $\beta$ is a weighted factor of $0.5 . \mathrm{Sa}=1.0$ serves as a threshold to delineate the combustion modes such that $\mathrm{Sa}$ less/greater than unity represents a strong/weak ignition.

To account for the turbulence effect, Im et al. [19] conducted a theoretical scaling analysis to derive a refined criterion to predict different ignition regimes a priori for an uniform mixture with turbulent velocity and temperature fluctuations. With an uniform composition, the gradient of ignition delay time, $\nabla \tau_{i g}$, is solely due to temperature variations, and the equation is expanded by a chain rule as

$$
\left|\nabla \tau_{i g}\right|=\left|\frac{d \tau_{i g}}{d T} \nabla T\right|
$$

where $d \tau_{i g} / d T$ represents the ignition delay sensitivity to temperature, and $\nabla T$ is the temperature gradient of the bulk mixture [19, 66].

In the RANS context, $\mathrm{Sa}$ is approximated as

$$
\mathrm{Sa} \approx \beta S_{L}\left|\frac{d \tau_{i g}}{d T}\right| \widetilde{\nabla T} \mid,
$$

where $S_{L}$ and $\tau_{i g}$ are evaluated using the initial bulk mixture conditions, $|\widetilde{\nabla T}|$ denotes the statistical mean temperature gradient. By performing the scaling analysis further, Im et al. [19] derived the following expression of the predicted Sankaran number as

$$
\mathrm{Sa}_{p}=K \mathrm{Da}_{l}^{-1 / 2}, \quad K=\beta \frac{1}{\left(\tau_{f} \tau_{i g}\right)^{1 / 2}}\left|\frac{d \tau_{i g}}{d T} T^{\prime}\right|
$$

$K$ and $T^{\prime}$ represent the normalized thermal ignition sensitivity, and the magnitude of thermal fluctuation, respectively. $\mathrm{Da}_{l}=\tau_{t} / \tau_{\text {ig }}$ is the integral scale Damköhler number defined as the ratio of the turbulence time scale to the ignition delay time. $\tau_{t}=l / u^{\prime}$ is the ratio of integral length scale to velocity fluctuation. $\tau_{f}=\alpha / S_{L}^{2}$ is the flame time scale, where $\alpha$ is the thermal diffusivity of the initial bulk fuel/air mixture.

Note that to distinguish with the exact Sankaran criterion in Eq. 2, the predicted Sankaran number in Eq. 5 is denoted as $\mathrm{Sa}_{p}$. Similar to $\mathrm{Sa}$, it is expected for a weak ignition if $\mathrm{Sa}_{p}>1$, and a strong ignition if $\mathrm{Sa}_{p}<1$.

\section{B. Ignition regime diagram with both temperature and concentration fluctuations}

The turbulent ignition regime diagram is further extended to account for both temperature and concentration fluctuations. With the present of both temperature and equivalence ratio fluctuations, $\nabla \tau_{i g}$ is expressed by a chain rule as

$$
\left|\nabla \tau_{\text {ig }}\right|=\left|\frac{\partial \tau_{i g}}{\partial T} \nabla T+\frac{\partial \tau_{i g}}{\partial \phi} \nabla \phi\right|
$$

where $\partial \tau_{i g} / \partial T$ and $\partial \tau_{i g} / \partial \phi$ are the partial derivatives of $\tau_{i g}$ with respect to temperature and equivalence ratio, which represent the ignition delay sensitivity to temperature and equivalence ratio, respectively. $\nabla T$ and $\nabla \phi$ are the temperature 
and equivalence ratio gradients of the bulk mixture [19]. Note that $\partial \tau_{i g} / \partial T$ and $\partial \tau_{i g} / \partial \phi$ are fuel-dependent property while $\nabla T$ and $\nabla \phi$ represent the bulk mixture property which varies for each specific operating condition.

In a real IC engine, depending on many factors including injection strategies, amount of EGR, intake charge heating, and wall heat loss, fuel properties, different $T-\phi$ distributions may occur at the TDC prior to the main auto-ignition event. Early direct injection may result in uncorrelated $T-\phi$ fields because of turbulent mixing and heat transfer. Late direct injection may produce negatively-correlated $T-\phi$ fields attributed to the evaporative cooling of fuel vaporization and incomplete mixing [23, 27, 29, 31, 68]. As a result, the temperature and equivalence ratio fields have a comparable integral length scale, $l_{T} \sim l_{\phi}$. A perfect negatively-correlated $T-\phi$ distribution yields $\nabla T=-\nabla \phi$, and an identical integral length scale, $l_{T}=l_{\phi}$ such that the $K$ factor is expressed as

$$
K=\beta \frac{1}{\left(\tau_{f} \tau_{i g}\right)^{1 / 2}}\left|\frac{\partial \tau_{i g}}{\partial T} T^{\prime}-\frac{\partial \tau_{i g}}{\partial \phi} \phi^{\prime}\right|
$$

In the previous DNS study [23, 24, 29], the scalar integral length scales were reasonably assumed to be identical, $l_{T}$ $=l_{\phi}$, for the uncorrelated $T-\phi$ cases. If there is no correlation between temperature and concentration fluctuations, both $T^{\prime}$ and $\phi^{\prime}$ contribute equally to increase $K$ regardless of the sign of $\partial \tau_{i g} / \partial T$ and $\partial \tau_{i g} / \partial \phi$ for the UC cases. Therefore the $K$ factor is written as

$$
K=\beta \frac{1}{\left(\tau_{f} \tau_{i g}\right)^{1 / 2}}\left(\left|\frac{\partial \tau_{i g}}{\partial T} T^{\prime}\right|+\left|\frac{\partial \tau_{i g}}{\partial \phi} \phi^{\prime}\right|\right)
$$

The generalized expression of the $K$ factor may be written as

$$
K=\beta \frac{1}{\left(\tau_{f} \tau_{i g}\right)^{1 / 2}}\left(\left|\frac{\partial \tau_{i g}}{\partial T} T^{\prime}\right|+s\left|\frac{\partial \tau_{i g}}{\partial \phi} \phi^{\prime}\right|\right)
$$

where $s$ is the sign of $(\nabla \widetilde{T} \cdot \nabla \widetilde{\phi})\left(\frac{\partial \tau_{i g}}{\partial T} \frac{\partial \tau_{i g}}{\partial \phi}\right)$ if there exists a correlation between $\nabla T$ and $\nabla \phi$, otherwise $s$ is positive regardless of the sign of $\frac{\partial \tau_{i g}}{\partial T} \frac{\partial \tau_{i g}}{\partial \phi}$.

\section{Deterministic criterion to quantify combustion intensity}

For a given initial condition of $T$ and $\phi$ fields of a DNS case, the spatial distribution of ignition delay and in turn exact $\mathrm{Sa}$ (Eq. 2) is computed for every grid point of the entire computational domain. From the exact Sa field, we propose $F_{\mathrm{S}, S}$ as a predictive metric of combustion intensity. $F_{\mathrm{S}, S}$ is defined as the volumetric fraction of Sa less than unity over the entire computational domain. As such $F_{\mathrm{Sa}, S}$ represents the contribution of strong ignition to the overall combustion process. $F_{\mathrm{Sa}, S}$ directly links with the heat release from the spontaneous ignition mode, $F_{S}$, which obtained from the Da-based analysis in the DNS studies [20, 22, 25, -30, 33].

The representative contour of $\tau_{i g}, \mathrm{Sa}$, and the corresponding PDF(Sa) for Case 8 is shown in Fig. 11-d, and Fig. 2 , respectively. The initial conditions of Case 6 (see Table 1) are $T_{0}$ of $900 \mathrm{~K}, \phi_{0}$ of $0.5, T^{\prime}$ of $30 \mathrm{~K}$, and $\phi^{\prime}=0.15$. The contribution of strong and weak ignition is visually depicted in Fig. 2 such that $F_{\mathrm{Sa}, S}$ can also be written as $F_{\mathrm{Sa}, S}=$ $\int_{0}^{1} P D F(\mathrm{Sa}) \mathrm{dSa}$. The contribution of the weak ignition (deflagration) is $F_{\mathrm{Sa}, \mathrm{W}}=1.0-F_{\mathrm{Sa}, S}$. If $F_{\mathrm{Sa}, S}\left(F_{\mathrm{Sa}, \mathrm{W}}\right)$ approaches unity, SI (WI) is dominant, otherwise a mixed combustion mode of SI and WI co-exists.

\section{Initial conditions}

Twelve 2-D DNS cases listed in Table 1 are simulated by varying three initial mean temperatures of $770 \mathrm{~K}, 900 \mathrm{~K}$, and $1045 \mathrm{~K}$ and two levels of mixture inhomogeneities, $T^{\prime}-\phi^{\prime}$ of $15 \mathrm{~K}-0.075$ and $30 \mathrm{~K}-0.15$. Both negatively-correlated (NC) and uncorrelated (UC) $T-\phi$ distribution are also examined. A 2-D computational domain of $3.2 \times 3.2 \mathrm{~mm}^{2}$ with $1280 \times 1280$ grids points was used for all DNSs. The corresponding grid resolution is $2.5 \mu \mathrm{m}$. This fine grid resolution is needed to resolve thin flame structures at high pressure. The DNS cases were performed on Shaheen, a 36 rack Cray XC40 system, at King Abdullah University of Science and Technology (KAUST).

The initial turbulent flow field is prescribed by an isotropic kinetic energy spectrum function of Passot and Pouquet [69] as the previous DNS studies [21, 23,-25, 27, 29, 30, 34, 41, 46, 70-73]. Initial fuel concentration and temperature fields are also generated by the same energy spectrum with different random numbers to reproduce thermal and compositional non-uniformities. For all DNSs, the most energetic length scale, $l_{e}$, of $1.0 \mathrm{~mm}$ is chosen. The 

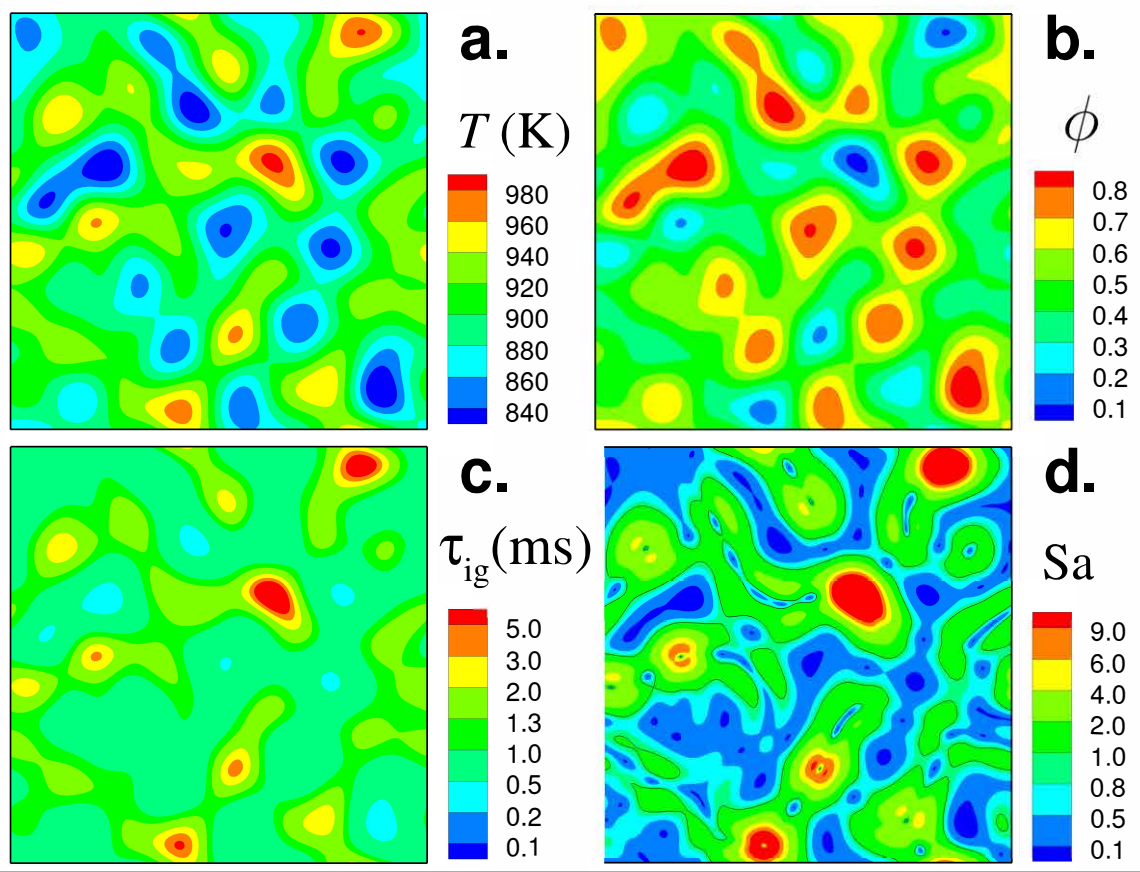

$\mathrm{Sa}$

Fig. 1 (a-b) Initial fields of temperature and equivalence ratio for Case 6 in Table 1 with $T_{0}-\phi_{0}$ and $T^{\prime}-\phi^{\prime}$ of $900 \mathrm{~K}-0.5$ and $30 \mathrm{~K}-0.15$, respectively, and (c-d) the corresponding ignition delay time and Sa distribution. The black iso-lines of $\mathrm{Sa}=1.0$ delineates two distinct regions of $\mathrm{Sa}<1$ and $\mathrm{Sa}>1$ which contribute to strong and weak ignition, respectively.

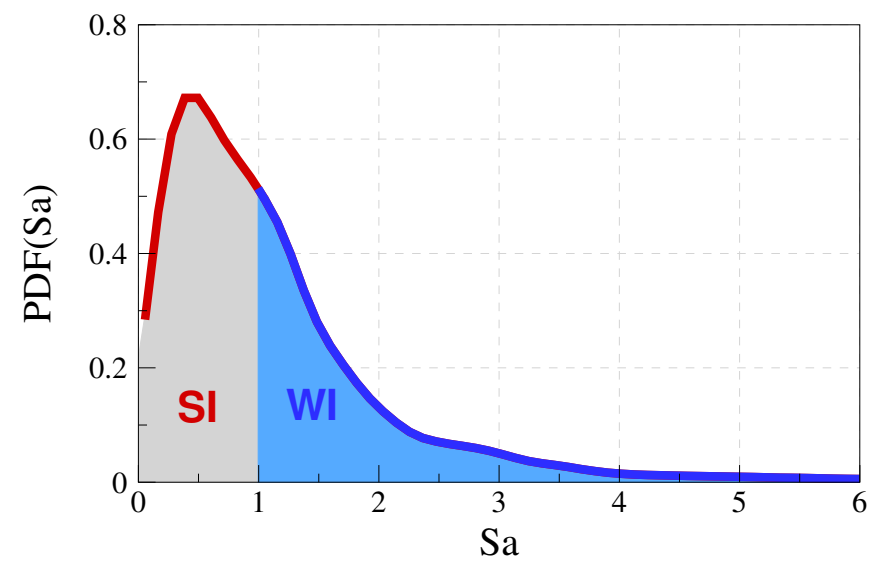

Fig. 2 A representative probability density function of Sa distribution, PDF(Sa). SI and WI denote strong ignition and weak ignition, respectively with a threshold of $S a=1$.

characteristic length scale of the concentration and temperature fields is selected identical to $l_{e}$. Turbulence intensity, $u^{\prime}$, of $1.0 \mathrm{~m} / \mathrm{s}$ is selected to ensure that the turbulence time scale, $\tau_{t}=l_{e} / u^{\prime}=1.0 \mathrm{~ms}$, is comparable to homogeneous $\tau_{i g}$ of $0.9 \mathrm{~m} / \mathrm{s}$. The nearly identical characteristic length scales and timescales for turbulence and scalar fields effectively enhance turbulence-chemistry interactions [22, 26]. The representative isocontours of initial field of temperature and equivalence ratio with a negative $T-\phi$ distribution for Case 6 in 1 are shown in Fig. 1 .

Dimethyl ether (DME) exhibiting a rich NTC behavior is chosen as a representative two-stage-ignition fuel to investigate the effect of temperature and equivalence ratio fluctuations on the combustion mode by varying the initial mean temperatures and different fluctuation levels. A 30-species non-stiff reduced mechanism for DME oxidation [74] 
was adopted as a diesel-like-fuel surrogate. The reduced mechanisms for DME oxidation were developed from the detailed mechanism consisting of 55 species and 290 elementary reactions. The reduced mechanism was validated under a wide range of equivalence ratio, pressure, and temperature conditions. Details of the reduced mechanisms for DME oxidation can be found in [74].

\begin{tabular}{ccccccccccccc}
\hline Case & Type & $\begin{array}{c}T_{0} \\
(\mathrm{~K})\end{array}$ & $\begin{array}{c}T^{\prime}(\mathrm{K}) \\
(\mathrm{K})\end{array}$ & $\phi^{\prime}$ & $\begin{array}{c}u^{\prime} \\
(\mathrm{m} / \mathrm{s})\end{array}$ & $\begin{array}{c}\tau_{t} \\
(\mathrm{~ms})\end{array}$ & $\begin{array}{c}S_{L} \\
(\mathrm{~m} / \mathrm{s})\end{array}$ & $\begin{array}{c}\mathrm{Da}_{l} \\
\begin{array}{c}\partial \tau_{i g} / \partial T \\
(\mathrm{~ms} / \mathrm{K})\end{array}\end{array}$ & $\begin{array}{c}\partial \tau_{i g} / \partial \phi \\
(\mathrm{ms})\end{array}$ & $K$ & $\mathrm{Sa}_{p}$ \\
\hline 1 & $\mathrm{NC}$ & 770 & 15 & 0.075 & 1.0 & 1.0 & 0.38 & 1.1 & $-8.7 \mathrm{E}-3$ & -1.14 & 0.16 & 0.1 \\
2 & $\mathrm{NC}$ & 770 & 30 & 0.150 & 1.0 & 1.0 & 0.38 & 1.1 & $-8.7 \mathrm{E}-3$ & -1.14 & 0.31 & 0.3 \\
3 & $\mathrm{UC}$ & 770 & 15 & 0.075 & 1.0 & 1.0 & 0.38 & 1.1 & $-8.7 \mathrm{E}-3$ & -1.14 & 0.74 & 0.7 \\
4 & $\mathrm{UC}$ & 770 & 30 & 0.150 & 1.0 & 1.0 & 0.38 & 1.1 & $-8.7 \mathrm{E}-3$ & -1.14 & 1.48 & 1.4 \\
5 & $\mathrm{NC}$ & 900 & 15 & 0.075 & 1.0 & 1.0 & 0.71 & 1.1 & $3.5 \mathrm{E}-3$ & -1.81 & 1.07 & 1.0 \\
6 & $\mathrm{NC}$ & 900 & 30 & 0.150 & 1.0 & 1.0 & 0.71 & 1.1 & $3.5 \mathrm{E}-3$ & -1.81 & 2.14 & 2.0 \\
7 & $\mathrm{UC}$ & 900 & 15 & 0.075 & 1.0 & 1.0 & 0.71 & 1.1 & $3.5 \mathrm{E}-3$ & -1.81 & 1.07 & 1.0 \\
8 & $\mathrm{UC}$ & 900 & 30 & 0.150 & 1.0 & 1.0 & 0.71 & 1.1 & $3.5 \mathrm{E}-3$ & -1.81 & 2.14 & 2.0 \\
9 & $\mathrm{NC}$ & 1045 & 15 & 0.075 & 1.0 & 1.0 & 1.17 & 1.1 & $-7.7 \mathrm{E}-3$ & -1.18 & 0.23 & 0.2 \\
10 & $\mathrm{NC}$ & 1045 & 30 & 0.150 & 1.0 & 1.0 & 1.17 & 1.1 & $-7.7 \mathrm{E}-3$ & -1.18 & 0.45 & 0.4 \\
11 & $\mathrm{UC}$ & 1045 & 15 & 0.075 & 1.0 & 1.0 & 1.17 & 1.1 & $-7.7 \mathrm{E}-3$ & -1.18 & 1.68 & 1.6 \\
12 & $\mathrm{UC}$ & 1045 & 30 & 0.150 & 1.0 & 1.0 & 1.17 & 1.1 & $-7.7 \mathrm{E}-3$ & -1.18 & 3.37 & 3.2 \\
\hline
\end{tabular}

Table 1 Physical parameters of the twelve 2-D DNS cases. The integral length scales are identical, $l_{e}=l_{T}=$ $l_{\phi}=1.0$ for all 2-D DNS cases. $\tau_{i g}$ denotes the time at which the maximum HRR occurs, the homogeneous $\mathrm{OD} \tau_{i g}$ is 0.9 for all the three $T_{0}$. NC and UC denote a negatively-correlated and uncorrelated $T-\phi$ distribution, respectively.
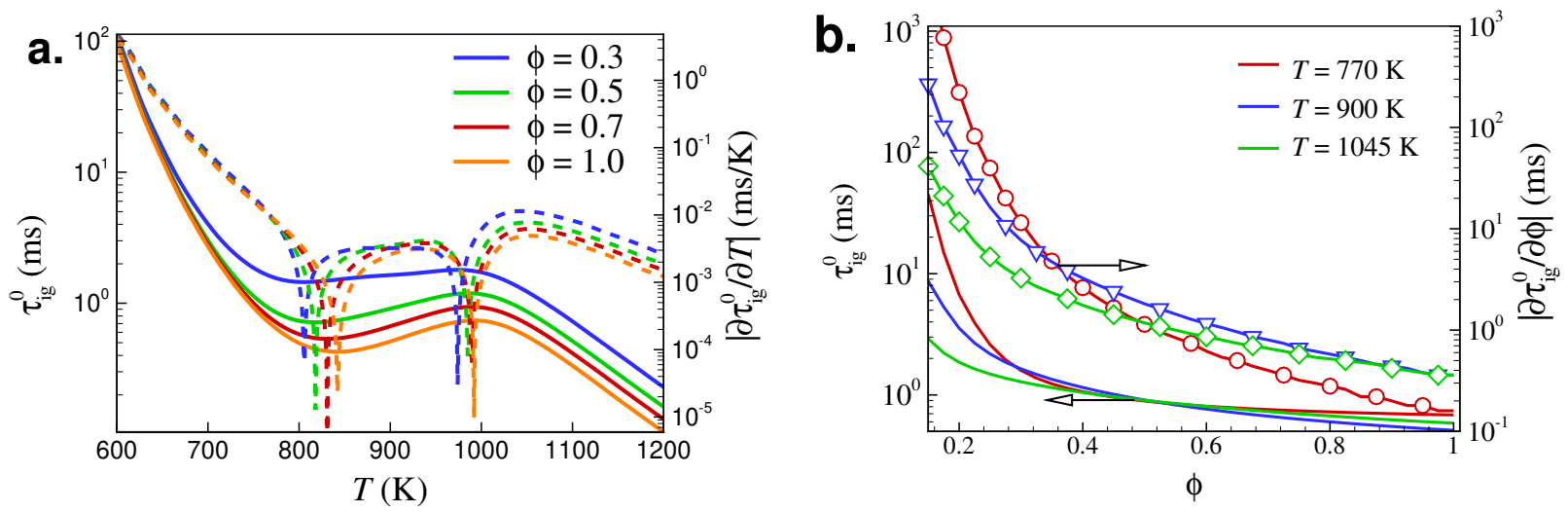

Fig. 3 (a) Homogeneous ignition delay of DME/air mixture as a function of temperature and equivalence ratio and its gradient with respect to temperature, $\partial \tau_{i g} / \partial T$, and (b) homogeneous ignition delay as a function of equivalence ratio and its gradient with respect to equivalence ratio, $\partial \tau_{i g} / \partial \phi$, for three different temperatures at equivalence ratio of 0.5 and a pressure of 30 atm.

Figure 3 shows the homogeneous ignition delay, $\tau_{i g}$, of DME/air mixture as a function of temperature and equivalence ratio for various $\phi_{0}$ and $T_{0}$ at $p_{0}=30$ atm. To compute $\mathrm{Sa}_{p}$ (Eq. 7 \& 8), the sensitivity of ignition delay to $T$ and $\phi$, $\partial \tau_{i g} / \partial T$ and $\partial \tau_{i g} / \partial \phi$, is needed. Their respective values at $T_{0}$ of $770 \mathrm{~K}, 900 \mathrm{~K}$, and $1045 \mathrm{~K}$ and $\phi_{0}=0.5$ are shown in Table 1. Two main points are noted from Fig. 3.

First, the ignition delay shows a non-monotonic behavior to temperature. In particular, $\partial \tau_{i g} / \partial T$ is positive at the low- and high-temperature regimes, whereas $\partial \tau_{i g} / \partial T$ is negative at the intermediate-temperature regime (i.e., from $830 \mathrm{~K}$ to $980 \mathrm{~K}$ in Fig. 3). Note that the absolute value of $\partial \tau_{i g} / \partial T$ is shown in Fig. 3. This regime is referred to as 
the negative-temperature coefficient (NTC) regime in which the ignition delay increases with increasing temperature. Because of the non-monotonic behavior of $\partial \tau_{i g} / \partial T$ - distinct from single-stage-ignition fuels, the variation of $\partial \tau_{i g} / \partial T$ within/near the NTC regime is marginal compared to those in the low- and high-temperature regimes in which $\partial \tau_{i g} / \partial T$ sharply decreases with increasing temperature. These features help explain the observed DNS results in [25, 29] that temperature fluctuation is less/more effective if $T_{0}$ is inside/outside the NTC regime. It is reported that the mixture within the NTC is more susceptible to autoignition. This behavior on the ignition mode is further quantified by $\mathrm{Sa}_{p}$ and $F_{\mathrm{S}, S}$ in the section V.A at different initial $T_{0}$.

Second, in contrast to the non-monotonic behavior of $\partial \tau_{i g} / \partial T, \partial \tau_{i g} / \partial \phi$ exhibits a monotonic behavior to equivalence ratio $-\partial \tau_{i g} / \partial \phi$ decreases with increasing $\phi$. As a result, the ratio of $\partial \tau_{i g} / \partial T$ to $\partial \tau_{i g} / \partial \phi, \frac{\partial \tau_{i g}}{\partial T} / \frac{\partial \tau_{i g}}{\partial \phi}$, shows a nonmonotonic behavior as $\partial \tau_{\text {ig }} / \partial T$-being negative/positive with $T_{0}$ inside/outside the NTC regime. Therefore, it is deduced from Eq. 7 that Sa increases (enhances deflagration) if $T_{0}$ inside the NTC regime $\left(\frac{\partial \tau_{i g}}{\partial T} / \frac{\partial \tau_{i g}}{\partial \phi}<0\right)$ while Sa decreases (promotes strong ignition) if $T_{0}$ outside the NTC $\left(\frac{\partial \tau_{i g}}{\partial T} / \frac{\partial \tau_{i g}}{\partial \phi}>0\right)$. It was found in the previous DNS results $[23,24,28-31]$ that a NC $T-\phi$ distribution attributed to the cooling effect of heat vaporization has a canceled-out effect on producing deflagration if $T_{0}$ outside the NTC regime while it has synergistic effect if $T_{0}$ inside the NTC regime. However, the deterministic prediction of the combustion modes by the extended $\mathrm{Sa}_{p}$ criterion applied for the NTC fuels for a wide range of $T_{0}$ at distinct temperature regimes together with different combinations of $T^{\prime}$ and $\phi^{\prime}$ levels merits further investigation.

\section{Results and discussion}

\section{A. Temperature fluctuations}

This section is devoted to considering temperature fluctuations only. The presence of both temperature and $\phi$ fluctuations are discussed in the next section. Three levels of $T^{\prime}$ are considered to verify the ignition criterion.

For a given initial field of temperature from a DNS case, exact Sa distribution is computed. Thus, the statistical Sa-based analysis including the statistical mean $\mathrm{Sa}, \overline{\mathrm{S}} \mathrm{a}$, and the volumetric fraction of $\mathrm{Sa}<1, F_{\mathrm{Sa}, S}$, is performed. Corresponding a $\bar{S}$ a number for each DNS case, a single predicted Sa number is also computed. Figure 4 shows the mean $\mathrm{Sa}$, predicted $\mathrm{Sa}$, and $F_{\mathrm{Sa}, S}$ as a function of initial mean temperatures.

Two main points are made from Fig. 4 . First, $\mathrm{Sa}_{p}$ accurately captures the combustion modes consistent with the previous DNS results [25- 27, 29]. As shown in Fig. 4 $T_{0}$ in/near the NTC regime is predicted to result in a strong ignition $\left(\mathrm{Sa}_{p}<1\right)$ even with $T^{\prime}$ up to 60 whereas a predominant mode of deflagration $\left(\mathrm{Sa}_{p}>1\right)$ is observed with a moderate $T^{\prime}$ for $T_{0}$ outside the NTC regime. The prediction shows satisfactory agreement with the DNS results in term of the maximum mean HRR, the HRR fraction from strong ignition from the Damköhler number analyses (not shown here). More specifically, Yoo et al. [25] found that with $T^{\prime}=60 \mathrm{~K}$, there are more than $93.4 \%$ heat release from spontaneous ignition (SI) at $T_{0}$ of $850 \mathrm{~K} \& 934 \mathrm{~K}$ while $62.3 \%$ heat release from SI at $T_{0}$ of $1008 \mathrm{~K}$. The corresponding predicted $\mathrm{Sa}_{p}$ of $n$-heptane [25] are $\mathrm{Sa}_{p}=0.33 \& 0.55(<1)$ at $T_{0}=850 \mathrm{~K} \& 933 \mathrm{~K}$, and $\mathrm{Sa}_{p}=1.60(>1)$ at $T_{0}=1008$ $\mathrm{K}$, corresponding to SI and WI, respectively.

Note that the prediction of $F_{\mathrm{Sa}, S}$ for the previous $n$-heptane and other fuels under various conditions [25- 34$]$ is validated by comparing $F_{\mathrm{Sa}, S}$ with $F_{S}$ of the previous DNS studies that shows a satisfactory agreement between $F_{\mathrm{Sa}, S}$ and $F_{S}$ (not shown here). For example, with $T^{\prime}=15 \mathrm{~K}$, both $F_{\mathrm{Sa}, S}$ and $F_{S}$ are approximately equal to unity for $T_{0}$ of 850 $\mathrm{K}, 934 \mathrm{~K}$, and $1008 \mathrm{~K}$, confirming a purely spontaneous ignition for all these three cases [25]. With $T^{\prime}=30 \mathrm{~K}, F_{\mathrm{Sa}, S}$ are $0.0,0.0$, and 0.92 for $T_{0}$ of $850 \mathrm{~K}, 934 \mathrm{~K}$, and $1008 \mathrm{~K}$, respectively, while $F_{S}$ from the Da-based analysis were found to be $0.98,0.99$, and 0.94 [25]. With $T^{\prime}=60 \mathrm{~K}, F_{\mathrm{Sa}, S}$ are $0.90,0.91$, and 0.66 for $T_{0}$ of $850 \mathrm{~K}, 934 \mathrm{~K}$, and $1008 \mathrm{~K}$, respectively, while $F_{S}$ were found to be $0.94,0.93$, and 0.62 [25]. Even with a high $T^{\prime}$ of $100 \mathrm{~K}, F_{\mathrm{Sa}, S}$ demonstrates a reliable prediction of $F_{S}\left(F_{\mathrm{Sa}, S}\right.$ and $F_{S}$ are $0.68 \& 0.63$ and $0.73 \& 0.62$ for $T_{0}$ of $850 \mathrm{~K}$ and $934 \mathrm{~K}$, respectively).

As shown in Fig. 4 the Sa-based prediction for DME/air mixtures is also found to be in line with the previous DNS results of $n$-heptane/air mixtures [25, 29] regardless of two different NTC fuels. Note that the selection of $T_{0}$ of $770 \mathrm{~K}$, $900 \mathrm{~K}$, and $1045 \mathrm{~K}$ in three different regimes with respect with the NTC regime is similar to those of Yoo et al. [25] and Luong et al. [29]. With $T^{\prime}$ increases up to $60 \mathrm{~K}$, in general, both $\mathrm{Sa}_{p}$ and $\overline{\mathrm{Sa}}$ is greater than unity for $T_{0}$ beyond the NTC regime (i.e., $T_{0}=700 \mathrm{~K}$ and $1100 \mathrm{~K}$ ), the predominant deflagration mode is expected to prevail; whereas for $T_{0}$ near/inside the NTC regime (i.e, $T_{0}$ in $770 \mathrm{~K}-1050 \mathrm{~K}$, strong ignition is more likely to be dominant since both predicted $\mathrm{Sa}_{p}$ and mean $\overline{\mathrm{Sa}}$ are less than unity and $F_{S}$ is approximately greater than 0.8 .

The second point to make is that $\mathrm{Sa}_{p}$ is able to reproduce the overall trend of $\overline{\mathrm{Sa}}$ (Fig. 4 - $\mathrm{a}$-b) except in the vicinity of 

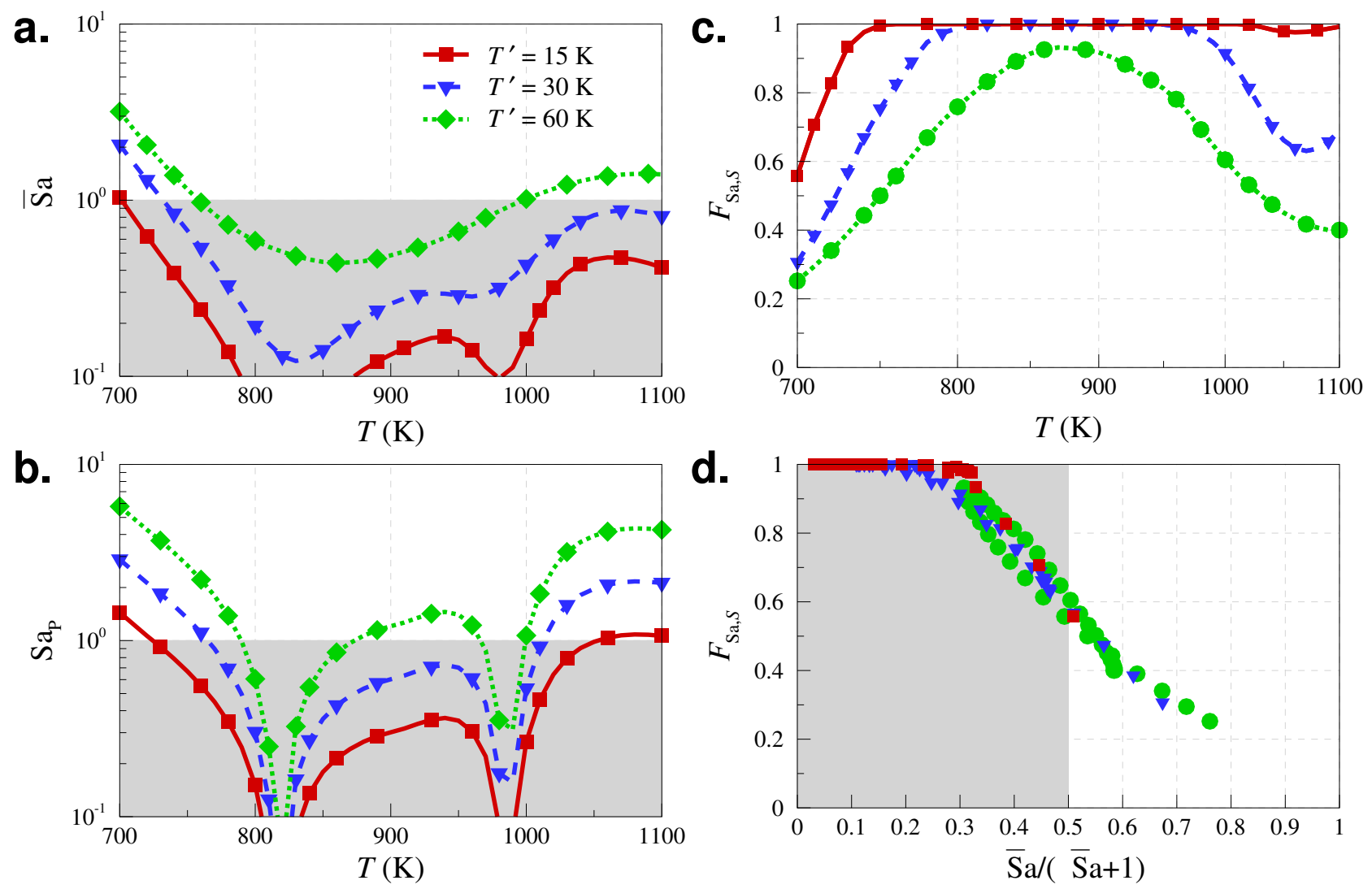

Fig. 4 (a-b-c) The mean $\mathrm{Sa}, \overline{\mathrm{S}} \mathrm{a}$, the predicted $\mathrm{Sa}, \mathrm{Sa}_{p}$, and the fraction of $\mathrm{Sa}<1, F_{\mathrm{S}, S}$, as a function of the initial mean temperature, and (d) $F_{\mathrm{Sa}, S}$ as a function of normalized $\overline{\mathrm{S}}$ a with different levels of temperature fluctuations for DME/air mixtures at $\phi_{0}=\mathbf{0 . 5}$.

the discontinuity regime caused by the NTC effect. The magnitude of $\mathrm{Sa}_{p}$ value is also comparable with the mean $\bar{S}$ a from the statistical Sa-based analysis. In addition, the figure $4 \mathrm{~d}$ shows a quantitative correlation between $\overline{\mathrm{S}} \mathrm{a}$ and $F_{\mathrm{Sa}, S}$ regardless of different $T_{0}$ and $T^{\prime}$. Note that the nearly-identical correlated pattern between $\overline{\mathrm{S}}$ a and $F_{\mathrm{Sa}, S}$ is also found for different fuel/air mixtures under various conditions (not shown here). Thank to the well-correlated $\overline{\mathrm{S}} \mathrm{a}-F_{\mathrm{Sa}, S}$ pattern, the magnitude of either $\overline{\mathrm{S}} \mathrm{a}$ or $\mathrm{Sa}_{p}$ can be used to estimate $F_{\mathrm{Sa}, S}$. With higher $\overline{\mathrm{S}}$ a corresponds to a lower $F_{\mathrm{Sa}, S}$, which translates into the lower combustion intensity as shown in Fig. $4 \mathrm{~d}$. With $\overline{\mathrm{Sa}}<1.0$, it is predicted that there is approximately more than $60 \%$ heat release contributed from the strong ignition mode. Otherwise, with $\bar{S} \mathrm{a}>1$, the weak ignition is predicted to be predominant. The deterministic $\mathrm{Sa}_{p}$-based prediction of combustion mode shows quantitative and quantitative agreement with previous DNS findings [19, 35, 41, 45, 66] such that $\mathrm{Sa}_{p}$ and $F_{\mathrm{Sa}, S}$ can be used as a reliable criterion.

\section{B. Temperature and concentration fluctuations}

The confident prediction of the mean $\overline{\mathrm{S}} \mathrm{a}, F_{\mathrm{Sa}, S}$, and $\mathrm{Sa}_{p}$ is further examined with the present of both $T^{\prime}$ and $\phi^{\prime}$ in this section. Note that equation 7 and Eq. 8 are used for the negatively-correlated and uncorrelated $T-\phi$ distribution, respectively.

Twelve 2-D DNS cases are performed with their physical parameters described in Table 1 The temporal evolution of the mean heat release rate (HRR) of all the twelve cases is shown in Fig. 5. Note that the cases with a high peak of the mean HRR and a narrow combustion duration correspond to predominant spontaneous combustion mode (SI) whereas a low peak of the mean HRR and a long combustion are attributed to greater contribution from deflagration (WI). Therefore it is expected that all the cases except Cases 4, 7-8, and 12 occurs in a strong-ignition mode whereas Cases 4, 7-8, and 12 are prevalent in the weak ignition mode. These observations are further verified by the probability 

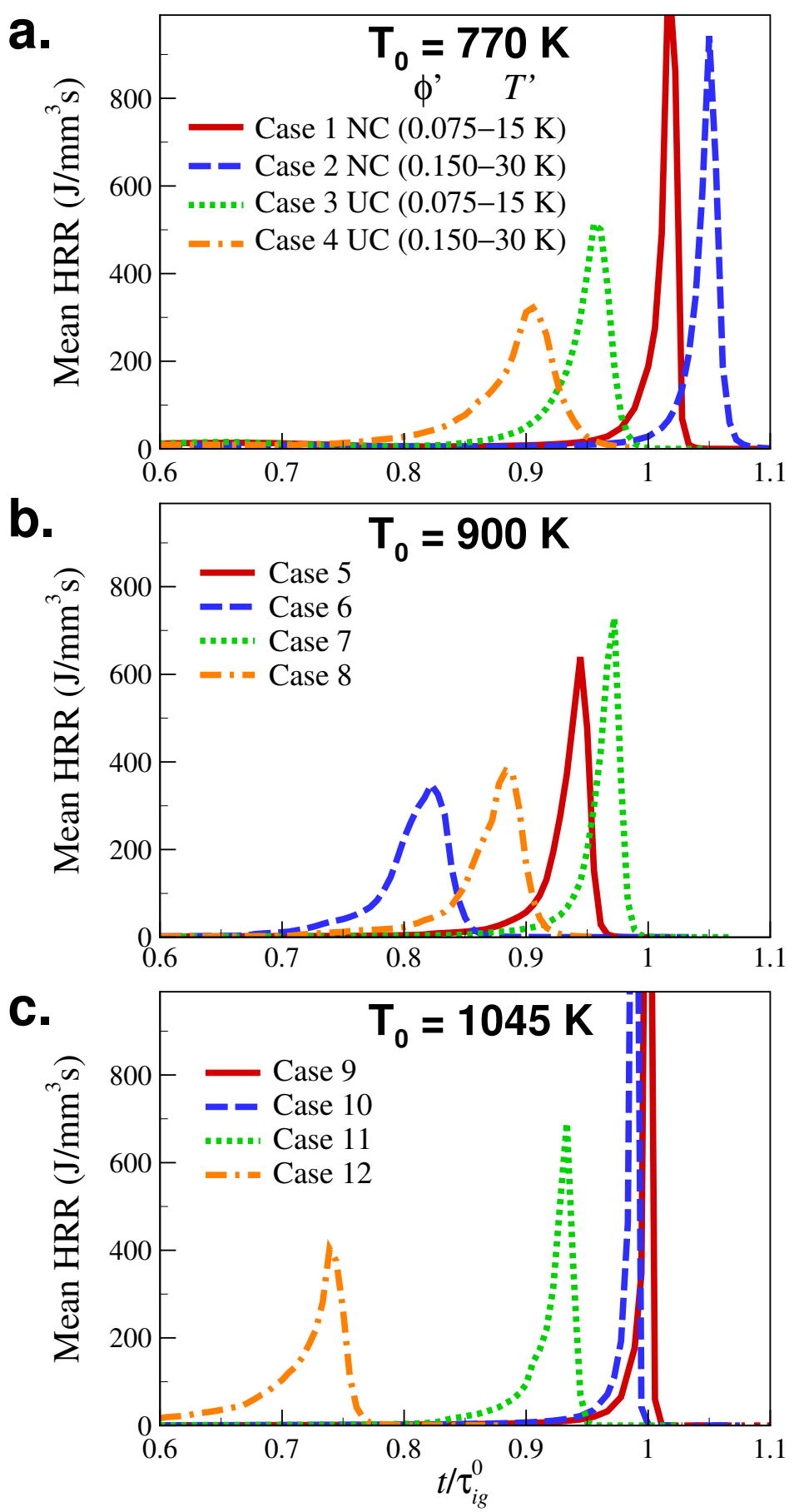

Fig. 5 The temporal evolution of the mean heat release rate (HRR) for the twelve 2-D DNS cases at three initial mean temperature, $T_{0}$ of $770 \mathrm{~K}, 900 \mathrm{~K}$, and $1045 \mathrm{~K}$ and two $T^{\prime}-\phi^{\prime}$ level of fluctuations of $15 \mathrm{~K}-0.075$ and $30 \mathrm{~K}-\mathbf{0 . 1 5}$. Both negative $T-\phi$ correlation (NC-the first two cases, 1-2, 5-6, and 9-10) and uncorrelated $T-\phi$ distribution (UC-the last two cases, 3-4, 7-8, and 11-12) are considered.

density function of $\mathrm{Sa}, \mathrm{PDF}(\mathrm{Sa})$.

Figure 6 shows that at a low level of fluctuation, $T^{\prime}-\phi^{\prime}$ of $15 \mathrm{~K}-0.075$, the span of PDF(Sa) is primarily distributed within a range of $\mathrm{Sa}<1$, indicating the most likely probability of strong ignition. On the contrary, a higher $T^{\prime}-\phi^{\prime}$ of 30 $\mathrm{K}-0.15$ the span of $\mathrm{PDF}(\mathrm{Sa})$ is more widely distributed for Case 6, the deflagration mode is expected to prevail; whereas 


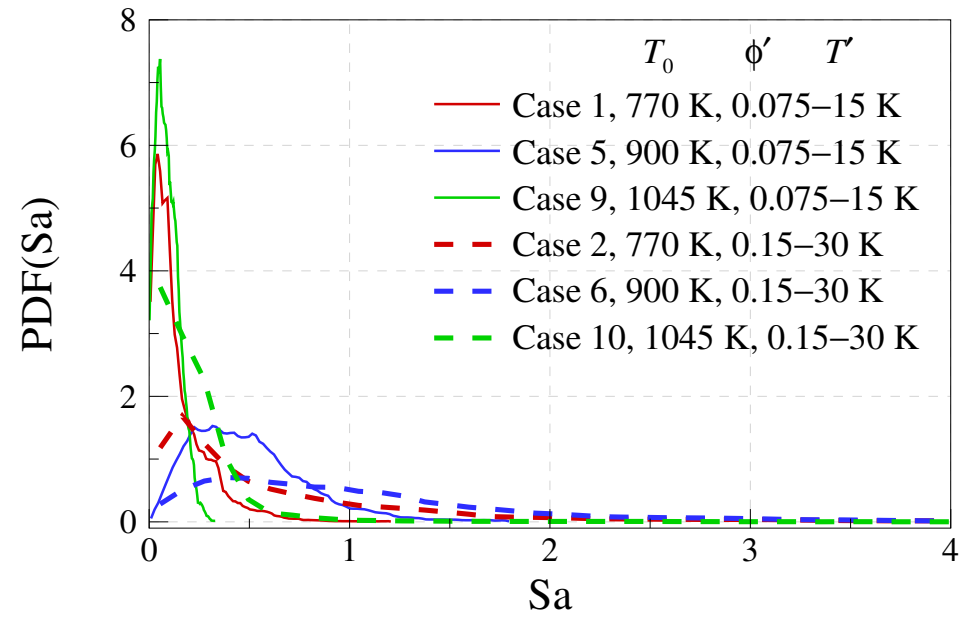

Fig. 6 The probability density function (PDF) of Sa, PDF(Sa), at three different initial mean temperatures of $770 \mathrm{~K}, 900 \mathrm{~K}$, and $1045 \mathrm{~K}$ with two $T^{\prime}-\phi^{\prime}$ level of fluctuations of $15 \mathrm{~K}-0.075$ (solid-thin lines) and $30 \mathrm{~K}-0.15$ (dashed-thick lines) (see Table 1 ).

for Cases $2 \& 10$, strong ignition is more likely to be dominant due to their narrow distribution of PDF(Sa) mostly spanned in the range of $\mathrm{Sa}<1$. The prediction based on the $\operatorname{PDF}(\mathrm{Sa})$ distribution shows an excellent agreement with the DNS results shown in Fig. 5 .

The quantitative prediction of the heat release from SI for these cases is also computed. $F_{\mathrm{Sa}, S}$ of Cases 1-5-9 and 2-6-1.0 are 0.99-0.94-1.0 and 0.80-0.56-0.97, respectively. The predicted Sa for twelve cases included in Table 1 is also in line with the DNS results. Most of the cases have $\mathrm{Sa}_{p}$ less than or close to unity except for Cases 4, 6, 8, and 12. The satisfactory agreement with the DNS results again verifies the reliably deterministic prediction of the $F_{\mathrm{Sa}, S}$ and $\mathrm{Sa} p$ metric.

\section{Conclusions}

The ignition regime diagram proposed by Im et al. [19] was extended to account for both temperature and concentration fluctuations. The autoignition modes of DME/air mixtures at the engine-relevant conditions with thermal and/or concentration non-uniformities were examined to validate the extended ignition criterion. The twelve 2-D DNS cases were performed to validate the predictive accuracy. It was demonstrated that the combustion modes of strong/weak ignition were well captured by the predicted Sankaran number, $\mathrm{Sa}_{p} . \mathrm{Sa}_{p}=1$ serves as a predictive metric to adequately delineate the transition between the weak and strong ignition regimes. In addition, the HRR fraction from strong ignition, $F_{S}$, representing the ignition intensity, is quantified a priori by the fraction of $\mathrm{Sa}<1, F_{\mathrm{Sa}, S}$ with a satisfactory agreement.

\section{Acknowledgments}

This work was sponsored by competitive research funding from King Abdullah University of Science and Technology. This research used the resources of the KAUST Supercomputing Laboratory (KSL).

\section{References}

[1] Dec, J. E., "Advanced compression-ignition engines-understanding the in-cylinder processes," Proc. Combust. Inst., Vol. 32, 2009, pp. 2727-2742.

[2] Lu, X., Han, D., and Huang, Z., "Fuel design and management for the control of advanced compression-ignition combustion modes," Prog. Energy Combust. Sci., Vol. 37, 2011, pp. 741-783.

[3] Saxena, S., and Bedoya, I. D., "Fundamental phenomena affecting low temperature combustion and HCCI engines, high load limits and strategies for extending these limits," Prog. Energy Combust. Sci., Vol. 39, 2013, pp. 457-488. 
[4] Dec, J. E., "Advanced Compression-Ignition Combustion for High Efficiency and Ultra-Low $\mathrm{NO}_{\mathrm{x}}$ and Soot," Encyclopedia of Automotive Engineering, edited by T. K. D. Crolla, D.E. Foster and N. Vaughan, John Wiley \& Sons, Ltd, 2014, pp. 1-40.

[5] Reitz, R. D., and Duraisamy, G., "Review of high efficiency and clean reactivity controlled compression ignition (RCCI) combustion in internal combustion engines," Prog. Energy Combust. Sci., Vol. 46, 2015, pp. 12-71.

[6] Paykani, A., Kakaee, A.-H., Rahnama, P., and Reitz, R. D., "Progress and recent trends in reactivity-controlled compression ignition engines," Int. J. Engine Res., 2016, pp. 481-524.

[7] Im, H. G., Arias, P. G., Chaudhuri, S., and Uranakara, H. A., "Direct Numerical Simulations of Statistically Stationary Turbulent Premixed Flames,” Combust. Sci. Technol., Vol. 188, 2016, pp. 1182-1198.

[8] Im, H. G., "Direct Numerical Simulations for Combustion Science: Past, Present, and Future," Modeling and Simulation of Turbulent Combustion, Springer, 2018, pp. 99-132.

[9] Bradley, D., Morley, C., Gu, X. J., and Emerson, D. R., "Amplified Pressure Waves During Autoignition: Relevance to CAI Engines,", No. 2002-01-2868, 2002. 00056.

[10] Gu, X., Emerson, D., and Bradley, D., "Modes of reaction front propagation from hot spots," Combust. Flame, Vol. 133, 2003, pp. 63-74.

[11] Gautam T Kalghatgi, and Derek Bradley, "Pre-ignition and 'super-knock' in turbo-charged spark-ignition engines," Int. J. Engine Res., Vol. 13, 2012, pp. 399-414.

[12] Kalghatgi, G. T., “The outlook for fuels for internal combustion engines,” Int. J. Engine Res., Vol. 15, 2014 , pp. 383-398.

[13] Kalghatgi, G. T., "Developments in internal combustion engines and implications for combustion science and future transport fuels," Proc. Combust. Inst., Vol. 35, 2015, pp. 101-115. 00072.

[14] Sow, A., Lee, B. J., Hern $\tilde{A}_{i}$ ndez PÃ@rez, F. E., and Im, H. G., "Detonation onset in a thermally stratified constant volume reactor," Proc. Combust. Inst., 2018, p. http://dx.doi.org/10.1016/j.proci.2018.08.043.

[15] Ali, M. J. M., Luong, M. B., Sow, A., Perez, F. H., and Im, H., "Probabilistic Approach to Predict Abnormal Combustion in Spark Ignition Engines," SAE paper, 2018, pp. 2018-01-1722.

[16] Jaasim, M., Sow, A., Perez, F. E., and Im, H. G., "A Computational Study of Abnormal Combustion Characteristics in Spark Ignition Engines," SAE paper, 2018, pp. 2018-01-0179.

[17] Peters, N., Kerschgens, B., and Paczko, G., "Super-Knock Prediction Using a Refined Theory of Turbulence," SAE Int. J. Engines, Vol. 6, 2013, pp. 953-967.

[18] Grogan, K. P., Scott Goldsborough, S., and Ihme, M., "Ignition regimes in rapid compression machines," Combust. Flame, Vol. 162, 2015, pp. 3071-3080.

[19] Im, H. G., Pal, P., Wooldridge, M. S., and Mansfield, A. B., "A Regime Diagram for Autoignition of Homogeneous Reactant Mixtures with Turbulent Velocity and Temperature Fluctuations,” Vol. 187, 2015, pp. 1263-1275.

[20] Sankaran, R., Im, H. G., Hawkes, E. R., and Chen, J. H., "The effects of non-uniform temperature distribution on the ignition of a lean homogeneous hydrogen-air mixture," Proc. Combust. Inst., Vol. 30, 2005, pp. 875-882.

[21] Chen, J. H., Hawkes, E. R., Sankaran, R., Mason, S. D., and Im, H. G., "Direct numerical simulation of ignition front propagation in a constant volume with temperature inhomogeneities: I. Fundamental analysis and diagnostics," Combust. Flame, Vol. 145, 2006, pp. 128-144.

[22] Hawkes, E. R., Sankaran, R., Pébay, P., and Chen, J. H., "Direct numerical simulation of ignition front propagation in a constant volume with temperature inhomogeneities: II. Parametric study," Combust. Flame, Vol. 145, 2006, pp. 145-159.

[23] Bansal, G., and Im, H. G., "Autoignition and front propagation in low temperature combustion engine environments," Combust. Flame, Vol. 158, 2011, pp. 2105-2112.

[24] Mittal, V., Cook, D., and Pitsch, H., “An extended multi-regime flamelet model for IC engines,” Combust. Flame, Vol. 159, 2012, pp. 2767-2776.

[25] Yoo, C. S., Lu, T., Chen, J. H., and Law, C. K., "Direct numerical simulations of ignition of a lean $n$-heptane/air mixture with temperature inhomogeneities at constant volume: Parametric study," Combust. Flame, Vol. 158, 2011, pp. 1727-1741. 
[26] Yoo, C. S., Luo, Z., Lu, T., Kim, H., and Chen, J. H., "A DNS study of ignition characteristics of a lean iso-octane/air mixture under and SACI conditions," Proc. Combust. Inst., Vol. 34, 2013, pp. 2985-2993.

[27] Luong, M. B., Luo, Z., Lu, T., Chung, S. H., and Yoo, C. S., "Direct numerical simulations of the ignition of lean primary reference fuel/air mixtures with temperature inhomogeneities," Combust. Flame, Vol. 160, 2013, pp. $2038-2047$.

[28] Luong, M. B., Lu, T., Chung, S. H., and Yoo, C. S., "Direct numerical simulations of the ignition of a lean biodiesel/air mixture with temperature and composition inhomogeneities at high pressure and intermediate temperature," Combust. Flame, Vol. 161, 2014, pp. 2878-2889.

[29] Luong, M. B., Yu, G. H., Lu, T., Chung, S. H., and Yoo, C. S., "Direct numerical simulations of ignition of a lean $n$-heptane/air mixture with temperature and composition inhomogeneities relevant to HCCI and SCCI combustion," Combust. Flame, Vol. 162, 2015, pp. 4566-4585.

[30] Luong, M. B., Yu, G. H., Chung, S. H., and Yoo, C. S., "Ignition of a lean PRF/air mixture under RCCI/SCCI conditions: A comparative DNS study," Proc. Combust. Inst., Vol. 36, 2017, pp. 3623-3631.

[31] Talei, M., and Hawkes, E. R., "Ignition in compositionally and thermally stratified $n$-heptane/air mixtures: A direct numerical simulation study," Proc. Combust. Inst., Vol. 35, 2015, pp. 3027-3035.

[32] Luong, M. B., Yu, G. H., Chung, S. H., and Yoo, C. S., "Ignition of a lean PRF/air mixture under RCCI/SCCI conditions: Chemical aspects," Proc. Combust. Inst., Vol. 36, 2017, pp. 3587-3596.

[33] Luong, M. B., Sankaran, R., Yu, G. H., Chung, S. H., and Yoo, C. S., "On the effect of injection timing on the ignition of lean PRF/air/EGR mixtures under direct dual fuel stratification conditions," Combust. Flame, Vol. 183, 2017, pp. 309-321.

[34] Kim, S. O., Luong, M. B., Chen, J. H., and Yoo, C. S., "A DNS study of the ignition of lean PRF/air mixtures with temperature inhomogeneities under high pressure and intermediate temperature," Combust. Flame, Vol. 162, 2015, pp. 717-726.

[35] Yang, Y., Dec, J., Dronniou, N., Sjöberg, M., and Cannella, W., "Partial Fuel Stratification to Control HCCI Heat Release Rates: Fuel Composition and Other Factors Affecting Pre-Ignition Reactions of Two-Stage Ignition Fuels," SAE Int. J. Engines, Vol. 4, 2011, pp. 1903-1920.

[36] Yang, Y., Dec, J. E., Dronniou, N., and Sjöberg, M., "Tailoring HCCI heat-release rates with partial fuel stratification: Comparison of two-stage and single-stage-ignition fuels," Proc. Combust. Inst., Vol. 33, 2011, pp. 3047-3055.

[37] Sjöberg, M., and Dec, J. E., "Smoothing Heat-Release Rates Using Partial Fuel Stratification with Two-Stage Ignition Fuels," SAE paper, 2006.

[38] Krisman, A., Hwakes, E. R., Kook, S., Sjöberg, M., and Dec, J. E., "On the potential of ethanol fuel stratification to extend the high load limit in stratified-charge compression-ignition engines," Fuel, Vol. 99, 2012, pp. 45-54.

[39] Yang, Y., Dec, J., Dronniou, N., and Cannella, W., "Boosted HCCI Combustion Using Low-Octane Gasoline with Fully Premixed and Partially Stratified Charges," SAE Int. J. Engines, 2012.

[40] Yu, R., and Bai, X.-S., "Direct numerical simulation of lean hydrogen/air auto-ignition in a constant volume enclosure," Combust. Flame, Vol. 160, 2013, pp. 1706-1716.

[41] El-Asrag, H. A., and Ju, Y., "Direct numerical simulations of exhaust gas recirculation effect on multistage autoignition in the negative temperature combustion regime for stratified HCCI flow conditions by using $\mathrm{H}_{2} \mathrm{O}_{2}$ addition," Combust. Theory Model., Vol. 17, 2013, pp. 316-334.

[42] El-Asrag, H. A., and Ju, Y., "Direct numerical simulations of $\mathrm{NO}_{\mathrm{x}}$ effect on multistage autoignition of DME/air mixture in the negative temperature coefficient regime for stratified HCCI engine conditions," Combust. Flame, Vol. 161, 2014, pp. 256-269.

[43] Bhagatwala, A., Lu, T., and Chen, J. H., "Direct numerical simulations of HCCI/SACI with ethanol," Combust. Flame, Vol. 161, 2014, pp. 1826-1841.

[44] Bansal, G., Mascarenhas, A., and Chen, J. H., "Direct numerical simulations of autoignition in stratified dimethyl-ether (DME)/air turbulent mixtures," Combust. Flame, Vol. 162, 2015, pp. 688-702.

[45] Bhagatwala, A., Sankaran, R., Kokjohn, S., and Chen, J. H., "Numerical investigation of spontaneous flame propagation under RCCI conditions," Combust. Flame, Vol. 162, 2015, pp. 3412-3426. 
[46] Zhang, F., Yu, R., and Bai, X. S., "Direct numerical simulation of PRF70/air partially premixed combustion under IC engine conditions," Proc. Combust. Inst., Vol. 35, 2015, pp. 2975-2982.

[47] Zhang, F., Yu, R., and Bai, X. S., "Effect of split fuel injection on heat release and pollutant emissions in partially premixed combustion of PRF70/air/EGR mixtures," Appl. Energy, Vol. 149, 2015, pp. 283-296.

[48] Tang, Q., Liu, H., Li, M., and Yao, M., "Study on the Double Injection Strategy of Gasoline Partially Premixed Combustion under a Light-Duty Optical Engine,” SAE Int. J. Engines, Vol. 9, 2016, pp. 2185-2193.

[49] Tang, Q., Liu, H., and Yao, M., "Simultaneous Measurement of Natural Flame Luminosity and Emission Spectra in a RCCI Engine under Different Fuel Stratification Degrees,” SAE Int. J. Engines, Vol. 10, 2017, pp. 2017-01-0714.

[50] Tang, Q., Liu, H., Li, M., Yao, M., and Li, Z., "Study on ignition and flame development in gasoline partially premixed combustion using multiple optical diagnostics," Combust. Flame, Vol. 177, 2017, pp. 98-108.

[51] Tang, Q., Liu, H., Li, M., Geng, C., and Yao, M., "Multiple optical diagnostics on effect of fuel stratification degree on reactivity controlled compression ignition," Fuel, 2017, pp. 688-698.

[52] Desai, S., Sankaran, R., and Im, H. G., "Unsteady deflagration speed of an auto-ignitive dimethyl-ether (DME)/air mixture at stratified conditions," Proceedings of the Combustion Institute, 2018, p. http://dx.doi.org/10.1016/j.proci.2018.09.019.

[53] Xu, C., Park, J.-W., Yoo, C. S., Chen, J. H., and Lu, T., "Identification of premixed flame propagation modes using chemical explosive mode analysis," Proc. Combust. Inst., 2018.

[54] Zhang, H., Hawkes, E. R., Chen, J. H., and Kook, S., "A numerical study of the autoignition of dimethyl ether with temperature inhomogeneities," Proc. Combust. Inst., Vol. 34, 2013, pp. 803-812.

[55] Pan, J., Wei, H., Shu, G., Chen, Z., and Zhao, P., "The role of low temperature chemistry in combustion mode development under elevated pressures," Combust. Flame, Vol. 174, 2016, pp. 179-193.

[56] Dai, P., Chen, Z., Chen, S., and Ju, Y., "Numerical experiments on reaction front propagation in n-heptane/air mixture with temperature gradient," Proc. Combust. Inst., Vol. 35, ????, pp. 3045-3052.

[57] Qi, C., Dai, P., Yu, H., and Chen, Z., "Different modes of reaction front propagation in n-heptane/air mixture with concentration non-uniformity,” Proc. Combust. Inst., Vol. 36, 2017, pp. 3633-3641.

[58] Dai, P., Qi, C., and Chen, Z., "Effects of initial temperature on autoignition and detonation development in dimethyl ether/air mixtures with temperature gradient," Proc. Combust. Inst., Vol. 36, 2017, pp. 3643-3650.

[59] Yu, H., and Chen, Z., "End-gas autoignition and detonation development in a closed chamber," Combust. Flame, Vol. 162, 2015, pp. 4102-4111.

[60] Pan, J., Shu, G., Zhao, P., Wei, H., and Chen, Z., "Interactions of flame propagation, auto-ignition and pressure wave during knocking combustion,” Combust. Flame, Vol. 164, 2016, pp. 319-328.

[61] Strozzi, C., Mura, A., Sotton, J., and Bellenoue, M., "Experimental analysis of propagation regimes during the autoignition of a fully premixed methane-air mixture in the presence of temperature inhomogeneities," Combust. Flame, Vol. 159, 2012, pp. 3323-3341.

[62] Walton, S. M., He, X., Zigler, B. T., Wooldridge, M. S., and Atreya, A., "An experimental investigation of iso-octane ignition phenomena," Combust. Flame, Vol. 150, No. 3, 2007, pp. 246-262.

[63] Mansfield, A. B., and Wooldridge, M. S., "High-pressure low-temperature ignition behavior of syngas mixtures," Combust. Flame, Vol. 161, 2014, pp. 2242-2251.

[64] Mansfield, A. B., Wooldridge, M. S., Di, H., and He, X., "Low-temperature ignition behavior of iso-octane," Fuel, Vol. 139, 2015, pp. 79-86.

[65] Pal, P., Mansfield, A. B., Arias, P. G., Wooldridge, M. S., and Im, H. G., "A computational study of syngas auto-ignition characteristics at high-pressure and low-temperature conditions with thermal inhomogeneities," Combust. Theory Model., Vol. 187, 2015, pp. 587-601.

[66] Pal, P., Valorani, M., Arias, P. G., Im, H. G., Wooldridge, M. S., Ciottoli, P. P., and Galassi, R. M., "Computational characterization of ignition regimes in a syngas/air mixture with temperature fluctuations," Proc. Combust. Inst., Vol. 36, 2017, pp. 3705-3716. 
[67] Zeldovich, Y. B., "Regime classification of an exothermic reaction with nonuniform initial conditions," Combust. Flame, Vol. 39, 1980, pp. 211-214.

[68] Hasegawa, R., and Yanagihara, H., "HCCI Combustion in DI Diesel Engine," SAE paper, 2003, pp. 2003-01-0745.

[69] Passot, T., and Pouquet, A., "Numerical simulation of compressible homogeneous flows in the turbulent regime," J. Fluid Mech., Vol. 118, 1987, pp. 441-466.

[70] Yoo, C. S., and Im, H. G., "Characteristic boundary conditions for simulations of compressible reacting flows with multidimensional, viscous and reaction effects," Combust. Theory Model., Vol. 11, 2007, pp. 259-286.

[71] Yoo, C. S., and Im, H. G., "Transient soot dynamics in turbulent nonpremixed ethylene-air counterflow flames," Proc. Combust. Inst., Vol. 31, 2007, pp. 701-708.

[72] Yoo, C. S., Sankaran, R., and Chen, J. H., "Three-dimensional Direct Numerical Simulation of a Turbulent Lifted Hydrogen Jet Flame in Heated Coflow: Flame Stabilization and Structure," J. Fluid Mech., Vol. 640, 2009, pp. 453-481.

[73] Yoo, C. S., Richardson, E. S., Sankaran, R., and Chen, J. H., “A DNS Study of a Turbulent Lifted Ethylene Jet Flame in Highly-Heated Coflow," Proc. Combust. Inst., Vol. 33, 2011, pp. 1619-1627.

[74] Bhagatwala, A., Luo, Z., Shen, H., Sutton, J. A., Lu, T., and Chen, J. H., "Numerical and experimental investigation of turbulent DME jet flames," Proc. Combust. Inst., Vol. 35, 2015, pp. 1157-1166. 